\title{
Phase equilibria and their applications
}

\author{
Ivo B. Rietveld ${ }^{\mathrm{a}}$ \\ Caractérisation des matériaux moléculaires à activité thérapeutique (CAMMAT), Faculté \\ de Pharmacie, Université Paris Descartes, 4 avenue de l'observatoire, 75006 Paris, France
}

Received 28 November 2016

Published online 18 April 2017

This special issue on phase behavior was instigated by the successful JEEP (Journées d'Étude des Équilibres entre Phases) meeting in March 2016 in Paris. The meeting is held yearly for over forty years and was established by a group of French researchers back in the seventies working on phase equilibria. It explains why its name is French even if the working language during the conference is nowadays English, as a large community from different countries participates (which has led to the proposition to call the conference 'Joint European days on Equilibria between Phases'). This special issue is not the proceedings of the conference, but it does demonstrate the vast research area in which phase equilibria are of importance.

Phase behavior can appear as something magical. To take a simple example, the freezing or melting of water occurs at constant temperature, which means that mixtures of water and ice can be used to control temperature very accurately. In first instance, and possibly even after one understands thermodynamics, this can appear a miracle.

An interesting question related to phase behavior is for example the preferential formation of one form of calcium carbonate by shellfish, whereas a more stable form of calcium carbonate exists. How could this happen? How does the animal control the formation of a less stable form? How can the animal prevent the interconversion into the more stable form? Similar questions exist in the pharmaceutical realm, where formulators need to ascertain the stability of a drug formulation, consisting for example of a powder, a tablet, or a suspension. A patient expects that his or her drug will work even after it has been stored on a shelf in the bathroom for over a year. This requires understanding of the solid-state behavior of drugs and mixtures of drugs and their sensitivity to water. Therefore, the FDA (Food and Drug Administration) and EMA (European Medicine Agency) require the pharmaceutical industry to prove that they can maintain their formulation for a given period of time.

Similar questions exist for metal tools in industry used under high pressure, strain, and high temperatures. Machinery cannot fail when working under demanding circumstances, as it can cause dangerous situations and it will result in loss of material and profit. Another interesting issue is the taste and mouth feel of chocolate, which highly depends on the proper crystal form of the cocoa butter. It possesses several different crystal structures, and the most sought after form melts just above $30{ }^{\circ} \mathrm{C}$ in

\footnotetext{
a e-mail: ivo.rietveld@parisdescartes.fr
} 
the mouth. Life itself also depends on phase behavior, as lipids that make up the cells can form different structures depending on their concentration in water. Self-assembly is for that matter driven by systems searching for equilibrium, even if they remain in a steady state or another non-equilibrium state.

Processes occur because they are driven towards equilibrium. Thus even if equilibrium is not the eventual goal of the experimenter or engineer (or life), understanding the equilibrium conditions of a system can help understand why a system changes in certain ways. An interesting example is the case of ritonavir. It is one of the first drug molecules that were found to be effective against HIV. Because of this, the formulation of the drug was carried out as quickly as possible and consisted of a suspension based on the only solid form that was found to exist during the development phase. Once the drug was on the market, it was observed that its effectiveness had dramatically decreased. Only after an intense research effort, the researchers understood that the absence of activity was caused by the crystallization of a more stable crystalline form that had not been previously known $[1,2]$. Due to this crystallization into a far less soluble form, the drug quantity taken up by the body did not exceed the necessary minimum concentration to be effective against HIV. Had the developers known, they could have prepared a formulation avoiding this crystallization in advance.

The scientific realization that systems tend to equilibrium only really started to gain prominence in the $18^{\text {th }}$ century with Amontons (1663-1705) in France discovering and contemplating an absolute zero in the temperature scale and with Black (1728-1799) in Great Britain studying latent heat of fusion of ice and the heat capacity of water and snow. It took hold in the $19^{\text {th }}$ century when the push for theoretical understanding and optimization of the first steam engines designed during the industrial revolution in England gave rise to the so-called mechanical theory of heat, the precursor of classical thermodynamics. A lot of work in this field was done in Europe by Carnot (the Carnot cycle) and Clapeyron (Clapeyron equation) in France and by Clausius (second law of thermodynamics) in Germany to name a few. In terms of phase equilibria, it was, however, the American Josiah Willard Gibbs (1839-1903) who gathered the available European knowledge by traveling to Great Britain and Germany and managed to distill and describe the essentials of modern day 'classical' thermodynamics used in phase equilibria and represented by the equation [3-5]:

$$
d U=T d S-P d V
$$

where $d U$ is the change of internal energy of the system under study depending on two variables, the change in entropy $d S$ of the system and the change in the volume of the system $d V$. In addition to this equation, classical thermodynamics depends on two postulates. 1) Energy in an isolated system is conserved and 2) for a spontaneous process, the entropy must always increase, reaching a maximum, when the system reaches equilibrium. Nernst added a third postulate: the entropy of a system at zero kelvin (or absolute zero) is equal to zero.

The work of Gibbs is the basis for the so-called "phase theory" - the thermodynamics of phase equilibria- even if at present researchers often prefer to use a Legendre transform of equation (1), which in the honor of J.W. Gibbs is called the Gibbs free energy:

$$
d G=-S d T+V d P .
$$

The advantage of this equation is that the variables of the Gibbs energy are the temperature and the pressure, variables that can be most easily controlled in a laboratory setting. Nonetheless, the original equation used by Gibbs should not be forgotten and it is shown in this special issue how both equations are related to each other and to two other representations of the internal energy, the enthalpy and the Helmholtz free energy $[6]$. 
The English researcher Maxwell, in line with Gibbs, developed the Maxwell equations relating the changes of different thermodynamic quantities with each other through partial derivatives [7] leading to a mathematical interpretation of thermodynamics. Dutch researchers, such as Van der Waals, were motivated by the work of J.W. Gibbs leading to the Van der Waals equation of state of a 'real' gas in contrast to the ideal gas. Bakhuis-Roozeboom continuing the graphical approach used by Gibbs proposed the only four possible pressure-temperature phase diagrams of crystalline dimorphism [8].

Fast forward to the current scientific situation, it is clear that the theoretical framework of thermodynamics developed in the second part of the $19^{\text {th }}$ century is well established. However, this framework only gives an overall macroscopic description of phase behavior, whereas the phase behavior of individual systems still cannot be satisfactorily described, even if computer calculations in the field of crystal structure predictions of organic molecules, for example, has made tremendous progress in the last decade [9]. Nonetheless, chemical systems and their phase behavior need to be studied experimentally either to improve descriptions of phases at the atomic level or due to general lack of data on the behavior of the system. Only in this way, can we increase our understanding and advance science to a point, where ultimately all phase behavior in materials can be described mathematically or at least simulated in the computer. One of the main objectives of this special issue is the exploration of experimental data and their comparison to theoretical approaches. It contains on the one hand phenomenological descriptions of the phase behavior of materials, be it organic or inorganic systems, and descriptions of equipment to obtain the necessary data and on the other hand theoretical descriptions in the form of theoretical approaches and simulations.

The importance of understanding phase behavior in materials becomes clear when one considers the incredible diversity of the subjects in this special issue. One of the more difficult fields is probably biological systems, which due to their complex makeup may not demonstrate a clearly defined equilibrium state. Many local minima in the internal energy may exist and in addition, living organisms maintain a steady state driven by potentials defined with respect to equilibrium states that are never reached. A simple and very applied example is presented here in relation to water vapor equilibria and biological matter concerning sorption isotherms of mint [10]. In this respect, it is also possible to use biological matter for absorption of other molecules, such as dyes [11].

Another applied issue in the realm of phase equilibria is that of water treatment and desalination [12], a subject that will only become more important with the increasing water scarcity in the world. Related to this subject are geochemical studies of the solubility of aluminium hydroxysulfates from aluminosilicate ores for processing applications [13].

Of course, many of the papers in this special issue belong to more traditional research areas of phase equilibria, such as that of metal systems, which have clear technological applications: the aluminium-barium phase diagram [14], local order in molten steel for control over its properties [15], glass-forming mixtures of metals [16], the holmium-germanium phase diagram [17], mixtures between a rare earth and gold for use in new technologies [18], the phase diagrams of Ag-In and $\mathrm{Cu}$-In [19], thermodynamic modeling of fluxing alloys of Ni-C-Cr-Si-B systems [20], and crystallization of $\mathrm{Al}-\mathrm{Co}-\mathrm{Dy}(\mathrm{Ho})$ amorphous alloys [21].

Another area that is industrially important is mixtures of small (often carbon containing) molecules, in particular in the petroleum industry. Understanding the phase behavior of such systems helps improving transport and storage of fuels as well as carbon capture. Examples in this special issue are theoretical approaches on liquid-vapor behavior in mixtures [22] and of argon [23]. Equipment to determine the 
vapor pressure of complicated mixtures has been described using a quartz crystal resonator [24].

Polymorphism in organic crystals is an important issue for the pharmaceutical and the food industry as some of the previous examples have illustrated. Experimental investigations of polymorphs are also important to provide the field of crystal structure calculation with the necessary experimental data for comparison and fine-tuning. In particular, the incorporation of the influence of temperature in the crystal structure calculations and accurate energy estimates are still difficult to carry out [25]. The relative stability between polymorphs can be investigated using differential scanning calorimetry and X-ray diffraction [26], with which also a pressure-temperature phase diagram can be determined as shown here for fluoxetine nitrate [27]. Related to the polymorphism issue is the solubility of pharmaceuticals, for which as is often the case nowadays algorithms are developed to predict solubility, which may facilitate the choice of the active molecule to develop [28].

One of the more challenging experimental questions concerning phase behavior is how the amorphous phase evolves into a stable crystal form and whether it does. In this respect, it is not clear whether the amorphous phase should be considered as a continuous series of 'metastable' states with a continuum of different thermodynamic properties or that one can even speak of poly-amorphism. Nonetheless, the amorphous (or viscous) phase allows the study of almost stationary molecules and can therefore be used in certain cases to investigate nucleation and local order. An example can be found in the paper on the mobility of $( \pm)$-methocarbamol [29]. Another example of how crystallization occurs in the amorphous phase has been given in an article on griseofulvin [30]. In this context, it is also important to be able to obtain the amorphous phase, so that its - crystallization - behavior can be studied. Equipment to obtain amorphous water and salt mixtures has been described in this special issue [31].

An important issue relevant to phase equilibria is the separation of racemic mixtures. Even if from an energetic point of view, both enantiomers have the same probability to crystallize, additives can be used to change the crystallization kinetics allowing only a single enantiomer to crystallize demonstrating a delicate interplay between thermodynamic equilibrium requirements and the kinetics of crystallization $[32]$.

For purification purposes, the phase diagrams of impurities with the main compound can be studied. An example is the binary phase diagrams of 9,10dihydroanthracene and carbazole with phenanthrene [33].

The formation of mixed crystals, like cocrystals and solvates, is an important subject, as in each case the conditions for formation and the resulting properties of the crystals are different. A very useful subject of investigation in this respect is the Buckminsterfullerene, which tends to form solvates with many different solvents [34].

In line with the graphical approach of J.W. Gibbs, it has been shown in this special issue how the different representations of the internal energy, $U, H, F$, and $G$ (respectively the internal energy itself, the enthalpy, the Helmholtz free energy and the Gibbs free energy) are graphically related to each other and how each of them can be related to characteristic experimental variables [6]. In relation to this rather theoretical treatise another contribution demonstrates how the combination of simple thermodynamic rules and graphical extrapolations allows one to obtain consistent topological pressure - temperature phase diagrams [35]. A similar approach is used to study the degrees of freedom in the melting of plastic crystals under pressure [36]. And an example of a temperature - volume diagram (a projection of the Helmholtz energy) can be found in the paper on ascorbic acid [37].

Finally, it can be shown that these thermodynamic approaches and phase diagrams, mainly applied to 'simple' matter can also be used for plasma [38]. 
It has been my pleasure to be the guest editor of this special issue on phase equilibria that really demonstrates how rich the research area of phase equilibria and its different applications is. I would therefore like to thank all the authors for their contributions, which make this special issue worth reading. However, I would like to thank in particular all the reviewers who while remaining anonymous have put a lot of work in giving their opinions about the papers in this special issue and whose work I never would have been able to do by myself. I hope in the end that the readers of this special issue will feel as enthusiastic as I do when reading this special issue on phase equilibria and its applications.

\section{References}

1. S.R. Chemburkar, J. Bauer, K. Deming, H. Spiwek, K. Patel, J. Morris, R. Henry, S. Spanton, W. Dziki, W. Porter, J. Quick, P. Bauer, J. Donaubauer, B.A. Narayanan, M. Soldani, D. Riley, K. McFarland, Org. Process Res. Dev. 4, 413 (2000)

2. J. Bauer, S. Spanton, R. Henry, J. Quick, W. Dziki, W. Porter, J. Morris, Pharm. Res. 18, $859(2001)$

3. J.W. Gibbs, Trans. Conn. Acad. 3, 108 (1875)

4. J.W. Gibbs, Trans. Conn. Acad. 3, 343 (1877)

5. J.W. Gibbs, The collected works of J.W. Gibbs - Volume 1: Thermodynamics (Green\&Co, NY, 1928)

6. R. Céolin, I.B. Rietveld, Eur. Phys. J. Special Topics 226, 1001 (2017)

7. The scientific papers of James Clerk Maxwell, edited by W.D. Niven (Cambridge University Press, Cambridge, 1890)

8. H.W. Bakhuis Roozeboom, Die heterogenen Gleichgewichte vom Standpunkte der Phasenlehre, Erstes Heft: Die Phasenlehre - Systeme aus einer Komponente (Friedrich Vieweg und Sohn, Braunschweig, 1901)

9. A.M. Reilly, R.I. Cooper, C.S. Adjiman, S. Bhattacharya, A.D. Boese, J.G. Brandenburg, P.J. Bygrave, R. Bylsma, J.E. Campbell, R. Car, D.H. Case, R. Chadha, J.C. Cole, K. Cosburn, H.M. Cuppen, F. Curtis, G.M. Day, R.A. DiStasio Jr., A. Dzyabchenko, B.P. van Eijck, D.M. Elking, J.A. van den Ende, J.C. Facelli, M.B. Ferraro, L. Fusti-Molnar, C.-A. Gatsiou, T.S. Gee, R. de Gelder, L.M. Ghiringhelli, H. Goto, S. Grimme, R. Guo, D.W. M. Hofmann, J. Hoja, R.K. Hylton, L. Iuzzolino, W. Jankiewicz, D.T. de Jong, J. Kendrick, N.J. J. de Klerk, H.-Y. Ko, L.N. Kuleshova, X. Li, S. Lohani, F.J. J. Leusen, A.M. Lund, J. Lv, Y. Ma, N. Marom, A.E. Masunov, P. McCabe, D.P. McMahon, H. Meekes, M.P. Metz, A.J. Misquitta, S. Mohamed, B. Monserrat, R.J. Needs, M.A. Neumann, J. Nyman, S. Obata, H. Oberhofer, A.R. Oganov, A.M. Orendt, G.I. Pagola, C.C. Pantelides, C.J. Pickard, R. Podeszwa, L.S. Price, S.L. Price, A. Pulido, M.G. Read, K. Reuter, E. Schneider, C. Schober, G.P. Shields, P. Singh, I.J. Sugden, K. Szalewicz, C.R. Taylor, A. Tkatchenko, M.E. Tuckerman, F. Vacarro, M. Vasileiadis, A. Vazquez-Mayagoitia, L. Vogt, Y. Wang, R.E. Watson, G.A. de Wijs, J. Yang, Q. Zhu, C.R. Groom, Acta Crystallogr. Sect. B 72, 439 (2016)

10. F. Taoufik, M. El Hadek, M. Chrif Hnini, M.B. Benchanaa, M. El Hammioui, L.M. Idrissi Hassani, Eur. Phys. J. Special Topics 226, 993 (2017)

11. R. Aziam, M. Chiban, H. Eddaoudi, A. Soudani, M. Zerbet, F. Sinan, Eur. Phys. J. Special Topics 226, 977 (2017)

12. M.G. Biyoune, A. Atbir, H. Bari, L. Hassnaoui, E. Mongach, A. Khadir, L. Boukbir, R. Bellajrou, M. Elhadek, Eur. Phys. J. Special Topics 226, 931 (2017)

13. A. Teyssier, V. Lagneau, J.M. Schmitt, J.J. Counioux, C. Goutaudier, Eur. Phys. J. Special Topics 226, 943 (2017)

14. H. Azza, N. Selhaoui, K. Achgar, Eur. Phys. J. Special Topics 226, 1143 (2017)

15. L. Son, V. Sidorov, Eur. Phys. J. Special Topics 226, 1091 (2017)

16. T. Kulikova, A. Maiorova, V. Bykov, Shunyaev, Eur. Phys. J. Special Topics 226, 1097 (2017) 
17. S. Kardellass, C. Servant, N. Selhaoui, Eur. Phys. J. Special Topics 226, 1075 (2017)

18. S. Otmani, R. Tamim, D. Moustaine, K. Mahdouk, Eur. Phys. J. Special Topics 226, $1123(2017)$

19. Z. Bahari, J. Dugué, Eur. Phys. J. Special Topics 226, 1137 (2017)

20. N. Ilinykh, A. Krivorogova, Eur. Phys. J. Special Topics 226, 1115 (2017)

21. V. Sidorov, S. Petrova, P. Svec Sr., P. Svec, D. Janickovic, A. Palitsina, Eur. Phys. J. Special Topics 226, 1107 (2017)

22. A. Abbaci, S. Ladjama, Eur. Phys. J. Special Topics 226, 957 (2017)

23. S. Ladjama, A. Abbaci, Eur. Phys. J. Special Topics 226, 967 (2017)

24. E. Orlandi, J.-L. Daridon, H. Carier, Eur. Phys. J. Special Topics 226, 1065 (2017)

25. Y.N. Heit, G.J.O. Beran, Acta Crystallogr. Sect. B 72, 514 (2016)

26. C.S.D. Lopes, C.E.S. Bernardes, M.F.M. Piedade, H.P. Diogo, M.E.M.D. Piedade, Eur. Phys. J. Special Topics 226, 849 (2017)

27. R. Céolin, I. Rietveld, Eur. Phys. J. Special Topics 226, 881 (2017)

28. B. Bouillot, T. Spyriouni, S. Teychené, B. Biscans, Eur. Phys. J. Special Topics 226, $913(2017)$

29. J.J. Moura Ramos, H.P. Diogo, Eur. Phys. J. Special Topics 226, 889 (2017)

30. J.F. Willart, E. Dudognon, A. Mahieu, M. Eddleston, M. Descamps, Eur. Phys. J. Special Topics 226, 837 (2017)

31. A.-A. Ludl, L. Bove, J. Li, M. Morand, S. Klotz, Eur. Phys. J. Special Topics 226, 1051 (2017)

32. P. Kongsamai, A. Maneedaeng, C. Flood, J.H. ter Horst, A.E. Flood, Eur. Phys. J. Special Topics 226, 823 (2017)

33. A. Burel, N. Couvrat, S. Tisse, Y. Cartigny, P. Cardinael, G. Coquerel, Eur. Phys. J. Special Topics 226, 869 (2017)

34. J. Ye, M. Barrio, P. Negrier, N. Qureshi, I.B. Rietveld, R. Céolin, J.-L. Tamarit, Eur. Phys. J. Special Topics 226, 857 (2017)

35. R. Ceolin, S. Toscani, I.B. Rietveld, M. Barrio, J.-L. Tamarit, Eur. Phys. J. Special Topics 226, 1031 (2017)

36. A. Aznar, P. Lloveras, M. Barrio, J.-L. Tamarit, Eur. Phys. J. Special Topics 226, 1017 (2017)

37. B. Nicolai, M. Barrio, J.-L. Tamarit, R. Ceolin, I.B. Rietveld, Eur. Phys. J. Special Topics 226, 905 (2017)

38. A.Y. Chigvintsev, I.L. Iosilevskiy, L.Y. Noginova, Eur. Phys. J. Special Topics 226, 1041 (2017) 